\title{
Acute respiratory distress syndrome in a case of diabetic ketoacidosis requiring ECMO support
}

\author{
Milad Darrat ${ }^{1}$, Brian Gilmartin'1, Carmel Kennedy ${ }^{1}$ and Diarmuid Smith ${ }^{1,2}$ \\ 'Department of Endocrinology and Diabetes, Beaumont Hospital, Dublin, Ireland and 2School of Medicine, Royal
} College of Surgeons in Ireland, Dublin, Ireland

Correspondence should be addressed

to M Darrat

Email

miladmustafa7@yahoo.com

\section{Summary}

Acute respiratory distress syndrome (ARDS) is a rare but life-threatening complication of diabetic ketoacidosis (DKA). We present the case of a young female, with no previous diagnosis of diabetes, presenting in DKA complicated by ARDS requiring extra corporeal membrane oxygenation (ECMO) ventilator support. This case report highlights the importance of early recognition of respiratory complications of severe DKA and their appropriate management.

\section{Learning points:}

- ARDS is a very rare but life-threatening complication in DKA.

- The incidence of ARDS remains unknown but less frequent than cerebral oedema in DKA.

- The mechanism of ARDS in DKA has multifactorial aetiology, including genetic predisposition.

- Early recognition and consideration of rare pulmonary complication of DKA can increase survival rate and provide very satisfactory outcomes.

- DKA patients who present with refractory ARDS can be successfully rescued by ECMO support.

\section{Background}

Acute respiratory distress syndrome (ARDS) is a very rare but life-threatening complication of diabetic ketoacidosis (DKA) (1). It is characterised by sudden onset progressive hypoxemia with bilateral pulmonary infiltrates on chest $\mathrm{x}$-ray (CXR) (1). The pathogenesis of ARDS in DKA remains unclear. It is thought that diabetic patients have a higher risk of capillary permeability, and in the presence of a severe metabolic acidosis, this permeability is further increased, leading to an increased volume of fluid in the alveolar space (2). However, it can be argued that many diabetic patients who present with severe metabolic acidosis do not develop ARDS (3). This suggests that the mechanism of ARDS in DKA is multifactorial in aetiology (4). Here we report a case of severe ARDS that required ECMO support in a previously healthy young female admitted to the intensive care unit (ICU) due to a new-onset severe DKA as the first presentation of her type 1 diabetes mellitus (DM).

\section{Case presentation}

A 29-year-old female was referred by her general practitioner to the emergency department (ED) during the SARS-CoV2 crisis with a 4-day history of vomiting, general malaise, atypical chest pain and epigastric pain. There was a recent history of polyuria and polydipsia over the preceding month. Her past medical history was unremarkable apart from mild eczema and a 3 pack-year smoking history. She was not on regular medications. On arrival to the ED, she was normotensive with a blood pressure of $141 / 100 \mathrm{mmHg}$ and a sinus tachycardia of 127 b.p.m., her temperature was 
low at $34.6^{\circ} \mathrm{C}$ with an oxygen saturation of $98 \%$ on room air and a respiratory rate of 22 breaths/min. The lungs, heart and lower limb examinations were all normal.

\section{Investigation}

On presentation, her arterial blood gas (ABG) revealed a severe high anion gap metabolic acidosis with an arterial $\mathrm{pH}$ of 6.87 and hyperglycaemia with a plasma glucose of $31.6 \mathrm{mmol} / \mathrm{L}$. Capillary ketones were $4.6 \mathrm{mmol} / \mathrm{L}$, bicarbonate was $2.9 \mathrm{mmol} / \mathrm{L}, \mathrm{PO}_{2} 11.7 \mathrm{kPa}$ and $\mathrm{PCO}_{2}$ $1.5 \mathrm{kPa}$. The CXR on admission was normal (Fig. 1). ECG revealed a sinus tachycardia but no evidence of acute coronary syndrome. Other initial laboratory tests showed a hypokalaemia of $3.2 \mathrm{mmol} / \mathrm{L}$, white cell count of $33.09 \times 10^{9} / \mathrm{L}$, neutrophils $28.43 \times 10^{9} / \mathrm{L}$, CRP of $13.9 \mathrm{mg} / \mathrm{L}$, normal plasma sodium and serum creatinine (Table 1 ).

\section{Treatment}

The patient was diagnosed with new-onset type $1 \mathrm{DM}$ with severe diabetic ketoacidosis. She commenced treatment for diabetic ketoacidosis (DKA) as per hospital protocol with immediate potassium replacement, fixed rate insulin infusion and volume expansion with crystalloids. Empiric treatment with intravenous amoxicillin/clavulanic acid was initiated. After initial resuscitation, the acidaemia and ketonaemia began to correct slowly ( $\mathrm{pH}$ 7.07, capillary ketones $4.1 \mathrm{mmol} / \mathrm{L}$ ) and the hypothermia resolved

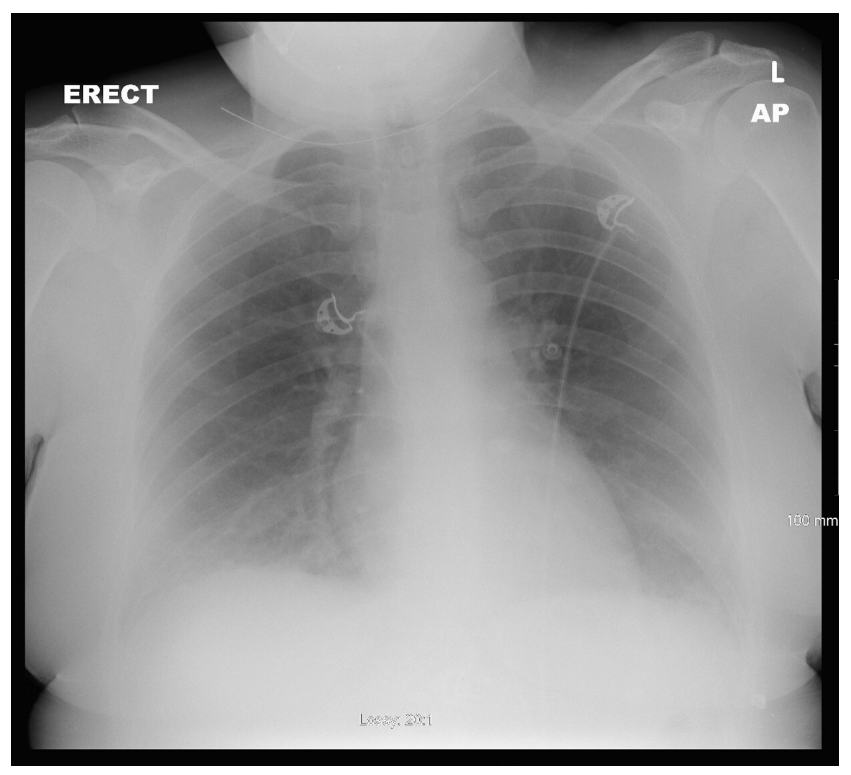

Figure 1

CXR on admission - heart, hilar and mediastinal contours are within normal limits. The lungs are clear. $\left(34.6-36.6^{\circ} \mathrm{C}\right)$. In the early hours of day 1 of presentation, the patient desaturated to $88 \%$ on room air and was commenced on supplemental oxygen. ABG on $3 \mathrm{~L}$ of oxygen $\left(\mathrm{FiO}_{2} 32 \%\right)$ showed a $\mathrm{PaO}_{2}$ of $6.4 \mathrm{kPa}, \mathrm{PCO}_{2} 2.3 \mathrm{kPa}$ and $\mathrm{pH} 7.24$ and bicarbonate of $7.5 \mathrm{mmol}$. She was initially treated as a suspected case of SARS-CoV2 and a nasopharyngeal swab was taken. She desaturated further with type 1 respiratory failure and a repeat CXR showed bilateral infiltrates consistent with ARDS-like (Fig. 2) picture. Her condition deteriorated with a further fall in her $\mathrm{pO}_{2}$ to $5.3 \mathrm{kPa}$ despite high flow oxygen via AIRVO $^{\text {m }} 2$ Humidification System, diuretic treatment, and continuation of antibiotics. She was transferred to the ICU where she was intubated and ventilated. In ICU she required an $\mathrm{FiO}_{2}$ of $100 \%$ with a peak end expiratory pressure of $15 \mathrm{~cm} \mathrm{H}_{2} \mathrm{O}$ to maintain a $\mathrm{PaO}_{2}$ of $10.3 \mathrm{kPa}$ and significant haemodynamic support with noradrenaline (40 $\mathrm{\mu g} / \mathrm{min})$ and vasopressin $(2.4$ units $/ \mathrm{h}$ ) required to maintain circulation. At that stage, the decision was made to proceed with lung protective ventilation in the form of venous-venous extracorporeal membrane oxygenation (ECMO) and Continuous VenoVenous Hemofiltration (CVVH). Two swabs taken $48 \mathrm{~h}$ apart and samples from a bronchoscopy while the patient was intubated were negative for SARS-CoV2.

Overnight on day 2 of ECMO her troponin level rose from 38 to $2869 \mathrm{ng} / \mathrm{L}$ (range reference of less than $14 \mathrm{ng} / \mathrm{L}$ ) in the presence of normal creatine kinase level of $84 \mathrm{U} / \mathrm{L}$ (range reference of 33-211 U/L). A bedside transthoracic echocardiogram (TTE) was suspicious for peri-myocarditis with a reduced ejection fraction of $45 \%$. Nonetheless, the patient's condition improved greatly on ECMO and the decision was made to discontinue ECMO after 4 days (Fig. 3). On day 7, the patient was extubated and maintained $\mathrm{PaO}_{2}$ of $16.7 \mathrm{kPa}$ on $\mathrm{FiO}_{2} 30 \%$ on AIRVO. Troponin remained elevated at $401 \mathrm{ng} / \mathrm{L}$ and a further echo on day 10 showed good left ventricle function with an ejection fraction of 50-55\% and a minimally thickened trileaflet aortic valve suggesting she may have had a stress myocarditis while on ECMO, which subsequently resolved. The insulin infusion used to control her blood glucose levels throughout her admission was discontinued on day 8 , and she was commenced on a basal/bolus regimen of subcutaneous insulin. Cultures of tracheal tube, urine and blood specimens remained all negative. CXR did not reveal neither cardiomegaly nor mediastinal lymphadenopathy. Also, the clinical course and rapid recovery did not seem to fit a viral, fungal or eosinophilic pneumonia. Hence, the definitive diagnosis was ARDS in association with severe DKA as the first manifestation of type $1 \mathrm{DM}$ in a 29-year-old lady. 
Table 1 Laboratory parameters during stay of admission.

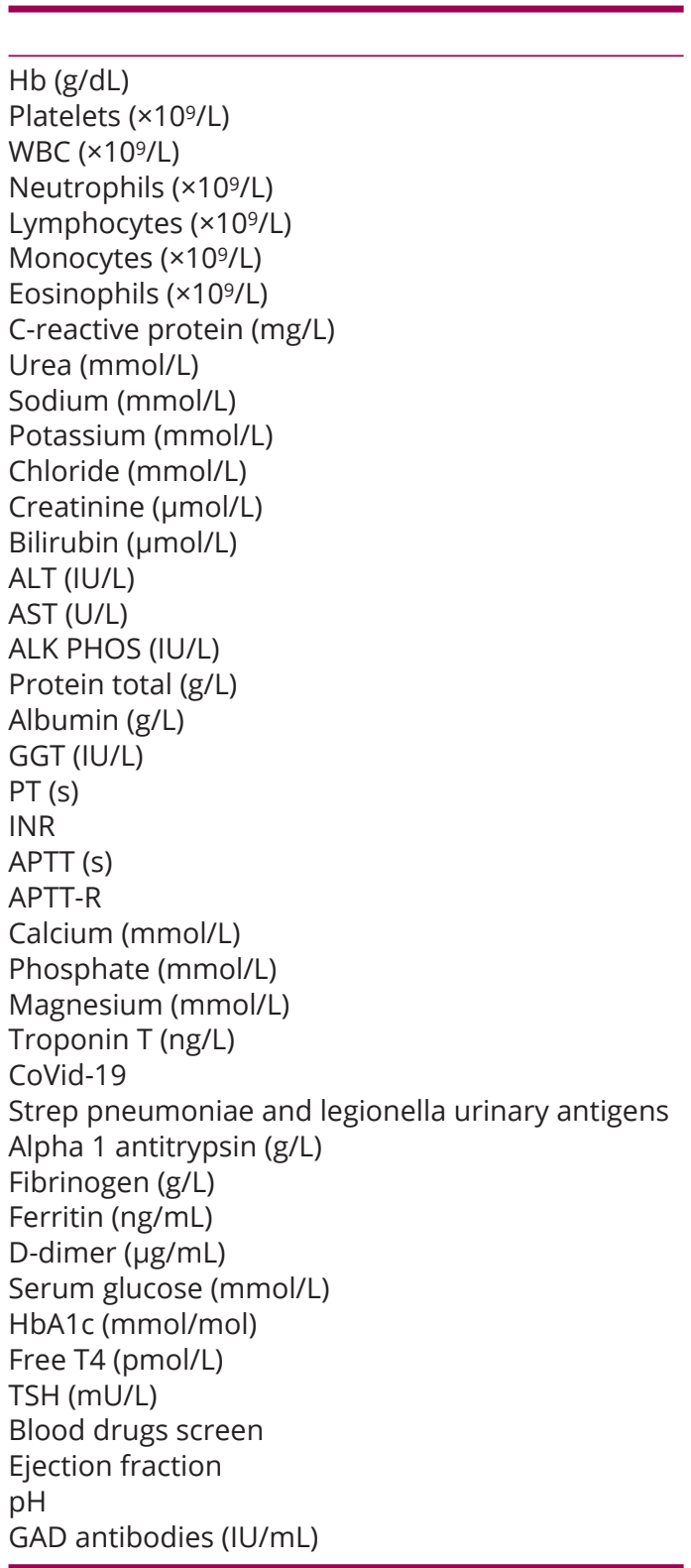

\section{Outcome and follow-up}

On day 11, she was discharged to the endocrine ward for further recovery and care. On day 16 , she was discharged home on a basal bolus regime of insulin novorapid and lantus. On follow-up in the outpatients 2 months later, she had a $\mathrm{HbA} 1 \mathrm{c}$ of $58 \mathrm{mmol} / \mathrm{mol}$ with strongly positive GAD antibodies consistent with her diagnosis of type 1 diabetes and normal cardiac function with a troponin of $<14 \mathrm{ng} / \mathrm{L}$. From the respiratory point of view, the patient remained well, with no shortness of breath, clear chest examination with $99 \%$ oxygen saturations on room air.

\begin{tabular}{|c|c|}
\hline ICU (day 1) & Post-extubation (day 11) \\
\hline 15.8 & 7.7 \\
\hline 304 & 260 \\
\hline 18.70 & 10.19 \\
\hline 16.98 & 6.69 \\
\hline 0.97 & 2.32 \\
\hline 0.51 & 0.75 \\
\hline 0.20 & 0.36 \\
\hline 116.5 & 18.9 \\
\hline 4.9 & 6.0 \\
\hline 142 & 132 \\
\hline 2.8 & 4.7 \\
\hline 113 & 99 \\
\hline 79 & 74 \\
\hline 9 & 4 \\
\hline 8 & 72 \\
\hline 26 & 57 \\
\hline 86 & 89 \\
\hline 56 & 60 \\
\hline 29 & 31 \\
\hline 13 & 70 \\
\hline 14.3 & 10.8 \\
\hline 1.20 & 0.91 \\
\hline 23.22 & 21.8 \\
\hline 0.72 & 0.68 \\
\hline 2.06 & 2.16 \\
\hline 0.12 & 1.07 \\
\hline 0.60 & 0.70 \\
\hline 29 & 17 \\
\hline Not detected & Not detected \\
\hline Not detected & - \\
\hline 1.38 & - \\
\hline 3.50 & - \\
\hline 321 & 1226 \\
\hline 3.32 & - \\
\hline 21.2 & 14 \\
\hline- & 65 \\
\hline - & 15.6 \\
\hline - & 2.20 \\
\hline- & - \\
\hline $45-50 \%$ & $50-55 \%$ (day 9) \\
\hline 7.22 & 7.37 \\
\hline- & 391 \\
\hline
\end{tabular}

\section{Discussion}

Here we report an unusual case of a young female patient who presented with new-onset DKA complicated by ARDS who required ICU admission and ECMO support. Other possible causes of ARDS, including sepsis, aspiration, inhalation injury, trauma, burns and SARS-CoV2, which are common causes of ARDS, were excluded (5).

The incidence of ARDS in DKA remains unknown, and it is usually occurring in adolescents and young adults (6). However, ARDS secondary to DKA is also described in the paediatric population (7). The mortality rate from DKA in 


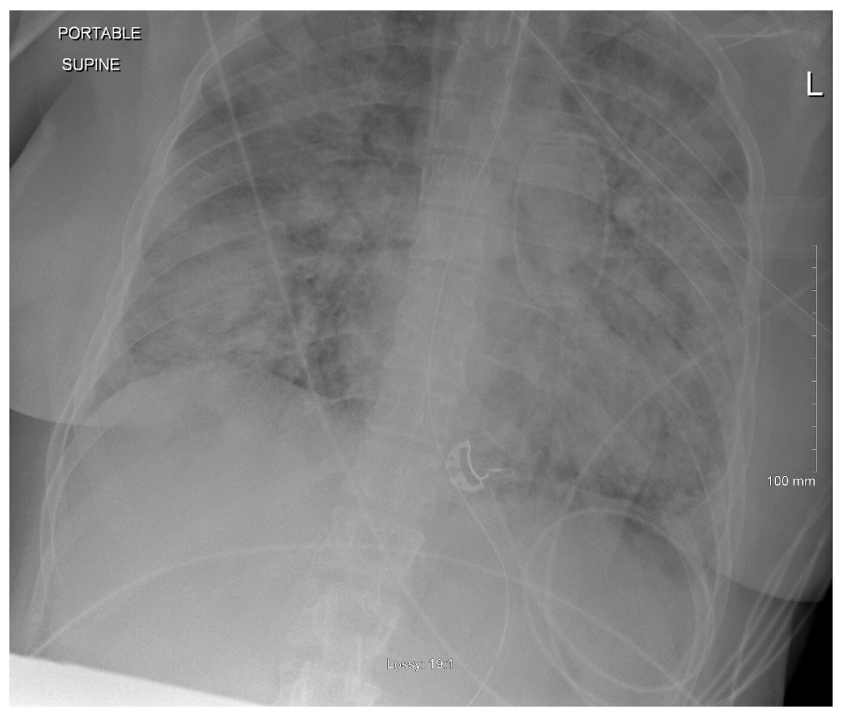

\section{Figure 2}

CXR while in ICU-support tubes and lines in a satisfactory position. There is extensive airspace consolidation throughout both lungs. No gross evidence of pleural effusion or pneumothorax.

children is around $0.30 \%$, and the major cause of all DKA deaths is cerebral oedema. ARDS also presents a predictor of high mortality but remains very rare in paediatric population (8).

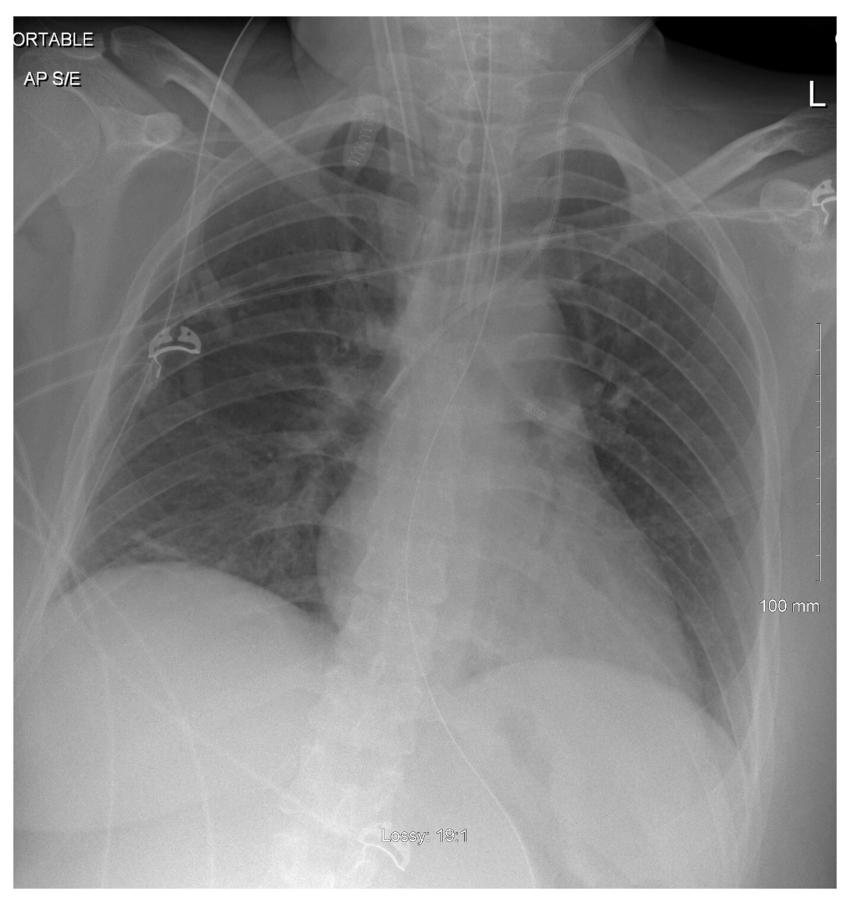

\section{Figure 3}

CXR post-ECMO - the lungs have substantially cleared in comparison to prior. There is some minimal plate atelectasis in the right lung base. Tubes and lines are in a good position.
This rare pulmonary complication does not yet have a well-defined time frame or clinical sequence. However, it usually occurs several hours after the initiation of DKA treatment similar to our patient presentation (9). The mechanism of ARDS in DKA remains poorly understood and likely multifactorial. The low $\mathrm{pH}$, as in our patient, could have precipitated pulmonary oedema by increasing pulmonary capillary permeability and altering the metabolism of alveolar surfactant $(10,11,12)$. This was also noticed in previous similar case reports (13). Nonetheless, many DKA patients present with very low $\mathrm{pH}$, yet do not develop ARDS. It is also suggested that aggressive fluid management could increase risk of ARDS by altering hydrostatic-oncotic pressures leading to pulmonary oedema, particularly in patients who already have the abnormal cardiac function on presentation (14, 15). Nevertheless, most DKA patients receive large volumes of intravenous fluid, but they do not end up with fluid overload. Furthermore, it is thought that severe metabolic changes in DKA can increase release of pro-inflammatory cytokines such as interleukin IL-1, IL-8 and TNF which lead to progression of the pulmonary infiltrates (16). Another proposed theory is genetic predisposition. Polymorphisms in surfactant associated protein B gene and angiotensin converting enzyme gene were found to be associated with susceptibility of ARDS $(4,17)$. This may play a role in identifying those at risk of ARDS or cerebral oedema in DKA, so a better individualised management plan approach can be initiated.

ECMO is a modified cardiopulmonary bypass circuit. It works as an artificial membrane lung and blood pump to provide gas exchange and systemic perfusion for patients with refractory cardiopulmonary instability. In a patient with isolated acute lung injury that does not need concurrent significant hemodynamic support, venovenous (VV) ECMO is the most common method used, as in our patient case, where blood was drained from a central vein, passed through a blood pump and oxygenator, and then returned to a central vein (18). The duration that a patient remains connected on ECMO is extremely variable as ECMO does not provide or accelerate lungs recovery. Nonetheless, it prevents further lung injury. The shortest successful ECMO usage for ARDS has been recorded was 48 $\mathrm{h}$ and in our patient case was 4 days (19).

ECMO is a costly, complex, and resource-intensive intervention that requires a well-trained and highly skilled multidisciplinary ICU team. Patients on ECMO can sustain a complex inflammatory reaction, similar to systemic inflammatory response syndrome (SIRS). ECMO may activate the complement, leukocyte, and pro-inflammatory 
coagulation pathways, which can lead to thrombosis or bleeding, the main complications associated with ECMO use (20). Fortunately, our patient had no complications from ECMO. To our knowledge, the use of ECMO has been reported in very few adult cases presenting with DKA. Our patient represents a unique successful example for the need to use this kind of novel therapy.

In conclusion, we report an unusual case of severe ARDS due to severe DKA in a young patient who required intensive care unit admission and ECMO ventilator support and survived without any significant sequels. This case report highlights the importance of early recognition and consideration of rare pulmonary complication of DKA and its appropriate management.

\section{Declaration of interest}

The authors declare that there is no conflict of interest that could be perceived as prejudicing the impartiality of the research reported.

\section{Funding}

This research did not receive any specific grant from any funding agency in the public, commercial or not-for-profit sector.

\section{Patient consent}

Written informed consent has been obtained from the patient for publication of the case report and accompanying images.

\section{Author contribution statement}

$M D$ and $B G$ wrote the draft of the manuscript. C K and D S reviewed the manuscript critically and approved its final version.

\section{References}

1 Thompson BT, Chambers RC \& Liu KD. Acute respiratory distress syndrome. New England Journal of Medicine 2017377 562-572. (https:// doi.org/10.1056/NEJMra1608077)

2 Holsclaw DS \& Torcato B. Acute pulmonary edema in juvenile diabetic ketoacidosis. Pediatric Pulmonology 199724 438-443. (https://doi. org/10.1002/(sici)1099-0496(199712)24:6<438::aid-ppul9>3.0.co;2-c)

3 Janz DR \& Ware LB. Approach to the patient with the acute respiratory distress syndrome. Clinics in Chest Medicine 201435 685-696. (https:// doi.org/10.1016/j.ccm.2014.08.007)

4 Chang L \& Li JG. Role of genetic factors in the development of acute respiratory distress syndrome. Journal of Translational Internal Medicine 20142 107-110. (https://doi.org/10.4103/2224-4018.141831)

5 ARDS Definition Task Force, Ranieri VM, Rubenfeld GD, Thompson BT, Ferguson ND, Caldwell E, Fan E, Camporota L \& Slutsky AS. Acute respiratory distress syndrome: the Berlin definition. JAMA $2012 \mathbf{3 0 7}$ 2526-2533. (https://doi.org/10.1001/jama.2012.5669)

6 Koves IH, Leu MG, Spencer S, Popalisky JC, Drummond K, Beardsley E, Klee K, Zimmerman JJ \& Diabetic Ketoacidosis Guideline Development Workgroup. Improving care for pediatric diabetic ketoacidosis. Pediatrics 2014134 e848-e856. (https://doi.org/10.1542/ peds.2013-3764)

7 Pavić I, Tješić Drinković D, Galić S, Tješić Drinković D \& Rojnić Putarek N. Acute respiratory distress syndrome in a four-year-old boy with diabetic ketoacidosis-case report. Acta Clinica Croatica 201857 588-592. (https://doi.org/10.20471/acc.2018.57.03.24)

8 Wolfsdorf JI, Allgrove J, Craig ME, Edge J, Glaser N, Jain V, Lee WW, Mungai LN, Rosenbloom AL, Sperling MA, et al. A consensus statement from the International Society for Pediatric and Adolescent Diabetes: diabetic ketoacidosis and hyperglycemic hyperosmolar state. Pediatric Diabetes 201415 (Supplement 20) 154-179. (https://doi.org/10.1111/ pedi.12165)

9 Karslioglu French E, Donihi AC \& Korytkowski MT. Diabetic ketoacidosis and hyperosmolar hyperglycemic syndrome: review of acute decompensated diabetes in adult patients. BMJ 201936511114. (https://doi.org/10.1136/bmj.11114)

10 Harris GD \& Fiordalisi I. Physiologic management of diabetic ketoacidemia. A 5-year prospective pediatric experience in 231 episodes. Archives of Pediatrics and Adolescent Medicine 1994148 1046-1052. (https://doi.org/10.1001/archpedi.1994.02170100044009)

11 Brun-Buisson CJL, Bonnet F, Bergeret S, Lemaire F \& Rapin M. Recurrent high permeability pulmonary edema associated with diabetic ketoacidosis. Critical Care Medicine 198513 55-56. (https:// doi.org/10.1097/00003246-198501000-00015)

12 Kumar B \& Ruff ST. Adult respiratory distress syndrome in diabetic ketoacidosis. Intensive Care Medicine 198410 269. (https://doi. org/10.1007/BF00256269)

13 Carroll P \& Matz R. Adult respiratory distress syndrome complicating severely uncontrolled diabetes mellitus: report of nine cases and a review of the literature. Diabetes Care 19825 574-580. (https://doi. org/10.2337/diacare.5.6.574)

14 Pischke MA. Diabetic ketoacidosis. Physician Assistant 200125 42-48.

15 Leonard RCF, Asplin C, McCormick CV \& Hockaday TD. Acute respiratory distress in diabetic ketoacidosis: possible contribution of low colloid osmotic pressure. BMJ 1983286 760-762. (https://doi org/10.1136/bmj.286.6367.760)

16 Hoffman WH, Burek CL, Waller JL, Fisher LE, Khichi M \& Mellick LB. Cytokine response to diabetic ketoacidosis and its treatment. Clinical Immunology 2003108 175-181. (https://doi.org/10.1016/s15216616(03)00144-x)

17 Tejera P, Meyer NJ, Chen F, Feng R, Zhao Y, O’Mahony DS, Li L, Sheu CC, Zhai R, Wang Z, et al. Distinct and replicable genetic risk factors for acute respiratory distress syndrome of pulmonary or extrapulmonary origin. Journal of Medical Genetics 201249 671-680. (https://doi.org/10.1136/jmedgenet-2012-100972)

18 Derwall M \& Roissant R. ECMO in severe acute respiratory distress syndrome: a light at the end of the tunnel? Lancet: Respiratory Medicine 20186 661-662. (https://doi.org/10.1016/S22132600(18)30278-9)

19 Kozinn J \& Wrisinger WC. ECMO for adults with severe respiratory failure. Missouri Medicine 2019116 58-62.

20 Millar JE, Fanning JP, McDonald CI, McAuley DF \& Fraser JF. The inflammatory response to extracorporeal membrane oxygenation (ECMO): a review of the pathophysiology. Critical Care 201620387. (https://doi.org/10.1186/s13054-016-1570-4)
Received in final form 12 May 2021

Accepted 15 June 2021 